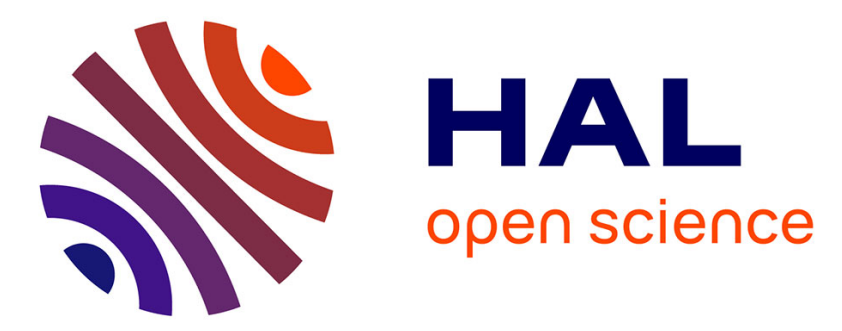

\title{
CHIP et cancers de l'ovaire : pour quelles patientes ?
}

Léonie Picard, Charlotte Bourgin, Frédérique Penault-Llorca, Marie Beguinot, Caroline Cornou, Guillaume Le Bouedec, Marie-Ange Mouret-Reynier, Frédéric Selle, Christophe Pomel

\section{- To cite this version:}

Léonie Picard, Charlotte Bourgin, Frédérique Penault-Llorca, Marie Beguinot, Caroline Cornou, et al.. CHIP et cancers de l'ovaire : pour quelles patientes ?. Bulletin du Cancer, 2019, 10.1016/j.bulcan.2019.04.003 . hal-02343537

\section{HAL Id: hal-02343537 \\ https://hal.science/hal-02343537}

Submitted on 21 Dec 2021

HAL is a multi-disciplinary open access archive for the deposit and dissemination of scientific research documents, whether they are published or not. The documents may come from teaching and research institutions in France or abroad, or from public or private research centers.
L'archive ouverte pluridisciplinaire HAL, est destinée au dépôt et à la diffusion de documents scientifiques de niveau recherche, publiés ou non, émanant des établissements d'enseignement et de recherche français ou étrangers, des laboratoires publics ou privés.

\section{다)(1) $(5$}

Distributed under a Creative Commons Attribution - NonCommercial| 4.0 International 


\section{CHIP et cancers de l'ovaire: pour quelles patientes?}

HIPEC in ovarian cancer: what should we expect?

PICARD Léonie ${ }^{1}$, BOURGIN Charlotte ${ }^{2}$, PENAULT-LLORCA Frédérique ${ }^{1}$, BEGUINOT Marie $^{1}$, CORNOU Caroline ${ }^{1}$, LE BOUEDEC Guillaume ${ }^{1}$, MOURET-REYNIER MarieAnge $^{1}$, SELLE Frédéric ${ }^{3}$, POMEL Christophe ${ }^{1 *}$,

1 - Centre Jean Perrin, 58 rue Montalembert, 63011 Clermont-Ferrand, France

2 - Institut de cancérologie de l'Ouest, 15 rue André Boquel, 49055 Angers, France

3 - Hôpital des Diaconesses, 12-18 rue du Sergent Bauchat, 75012 Paris, France

* Pour correspondance: Christophe POMEL, Centre Jean Perrin, 58 rue Montalembert, 63011 Clermont-Ferrand, France mail : christophe.pomel@ clermont.unicancer.fr 


\section{Résumé}

Une étude publiée en 2018 dans le New England Journal of Medicine suggère que la chimiothérapie intra-péritonéale hyperthermique (CHIP) apporte un bénéfice aux patientes présentant une carcinose péritonéale d'origine ovarienne, opérées après chimiothérapie néoadjuvante. Ces résultats contrastent avec ceux de l'étude PRODIGE 7, qui remettent en question la CHIP dans les carcinoses péritonéales d'origine colique. Le message apparaît dans un contexte où les thérapies ciblées constituent de nouveaux standards dans le traitement des carcinoses péritonéales d'origine ovarienne en première intention ou en situation de rechute. Dans cette publication, nous cherchons à faire le point de l'intérêt potentiel de la CHIP dans cette indication.

Mots-clés : CHIP, ovaire, carcinose péritonéale HIPEC, ovarian, carcinomatosis

\section{Abstract}

Results of PRODIGE 7 study demonstrate that the use of HIPEC is not beneficial for patients in the treatment of colorectal carcinomatosis. Nevertheless, a recent study published in New England Journal of Medicine showed that hyperthermic intra-peritoneal chemotherapy (HIPEC) increased overall survival for patients with ovarian peritoneal carcinomatosis. Although, the emergence of targeted therapies (anti-angiogenic agents, PARP-inhibitors, antiPDL1) results in new standards of treatment in first line or recurrence disease. In this general context, what is the potential interest of HIPEC for the treatment of ovarian carcinoma?

Keywords: HIPEC, ovarian, carcinomatosis

\section{Introduction.}

Avec près de 4700 nouveaux cas estimés en 2017 en France, le cancer de l'ovaire se situe au cinquième rang des cancers féminins [1]. Dans deux-tiers des cas le cancer de l'ovaire est diagnostiqué à un stade avancé, avec présence d'une carcinose péritonéale. Son histoire naturelle est marquée par des récidives péritonéales dans $70 \%$ des cas à partir du stade FIGO III [2]. La stratégie thérapeutique actuelle de la carcinose péritonéale d'origine ovarienne, associe une chimiothérapie systémique à une chirurgie de cytoréduction complète, lorsque la maladie est résécable. La place de la chimio-hyperthermie intrapéritonéale (CHIP), 
admise dans le traitement des carcinoses péritonéales d'origine non gynécologique jusqu'alors, reste discutée dans le cadre du traitement des carcinoses péritonéales ovariennes. Le péritoine constitue le site préférentiel de dissémination et de rechute du cancer de l'ovaire, c'est pourquoi l'administration de produits cytotoxiques directement dans la cavité péritonéale est une voie de traitement pertinente [3]. Elle permet d'obtenir localement de fortes concentrations médicamenteuses : dix à mille fois supérieures à celles du plasma, en comparaison à chimiothérapie intraveineuse, et la barrière péritonéo-plasmatique permet de limiter la toxicité systémique [4,5]. Deux techniques ont été mises au point : la chimiothérapie intrapéritonéale, grâce à un cathéter mis en place dans la cavité péritonéale, avec des administrations de chimiothérapie itératives en post-opératoire, et la CHIP, administrée en une fois, en fin de procédure chirurgicale, et associée à de l'hyperthermie.

Trois essais randomisés du GOG ont en effet montré que l'association d'une chimiothérapie intraveineuse et intrapéritonéale, après chirurgie de cytoréduction complète, prolonge la survie globale de 17 mois des patientes atteintes d'un cancer de l'ovaire de stade FIGO III [68]. Cependant, la répartition des produits cytotoxiques dans la cavité péritonéale est hétérogène, et la pénétration tissulaire des produits en profondeur est limitée à quelques millimètres [9]. De plus, ce gain de survie est obtenu au prix d'une toxicité supérieure de la voie intrapéritonéale à celle de l'intraveineuse, responsable d'une altération de la qualité de vie: les difficultés de tolérance, en particulier en regard des cathéters intra-péritonéaux, conduisent ainsi à un abandon de la voie intrapéritonéale dans près de la moitié des cas [10,11]. La CHIP présente l'avantage de traiter la maladie péritonéale résiduelle, au moment où la charge tumorale est la plus faible, dans un abdomen libre de toutes adhérences. Les tissus tumoraux sont plus thermosensibles que les tissus sains, et l'effet cytotoxique est potentialisé à $40^{\circ} \mathrm{C}$ [12]. L'hyperthermie favorise la pénétration des produits de chimiothérapie dans le péritoine et augmente la chimiosensibilité des cellules tumorales, grâce 
à un blocage des mécanismes de réparation de l'ADN, une inhibition de l'angiogenèse, une exposition de récepteurs reconnus par les lymphocytes Natural Killer, et une induction de l'apoptose [13]. Les complications spécifiques liées à la CHIP sont essentiellement d'ordre hématologiques, et sont dominées par le risque d'insuffisance rénale liée à l'utilisation prépondérante du Cisplatine [14]. Cependant, comparativement à la chimiothérapie intrapéritonéale, les effets indésirables systémiques sont moindres, du fait d'une administration unique et limitée dans le temps.

\section{Quelle place pour la CHIP en 2019 ?}

En 2017, deux études prospectives présentées à l'ASCO, dont l'une a été publiée dans le New England Journal Of Medicine, posent la question de la place de la CHIP dans le traitement des carcinoses péritonéales ovariennes [15,16].

L'étude de phase III OVHIPEC, publiée par van Driel et al. [16] évalue la place de la CHIP en chirurgie intervallaire chez des patientes présentant une néoplasie ovarienne ou tubaire avec atteinte péritonéale initiale (stade FIGO III), non résécables d'emblée. La CHIP à base de Cisplatine $(100 \mathrm{mg} / \mathrm{m} 2)$ est réalisée en circuit fermé avec 90 minutes de perfusion. Deux cent quarante-cinq patientes ont été incluses, 123 dans le bras sans CHIP (CHIP-), 122 dans le bras avec CHIP (CHIP+). En intention de traiter, $89 \%$ des patientes CHIP- et $81 \%$ des patientes CHIP+ ont présenté une récidive $(\mathrm{p}=0,003)$. La survie sans récidive était plus longue de trois mois et demi dans le bras CHIP+ comparé au bras CHIP- (14,2 mois versus 10,7 mois ; IC $95 \%$ 0,50-0,87 ; HR : 0,66 ; p=0,003). La survie globale était de 33,9 mois dans le bras CHIP- et de 45,7 mois dans le bras CHIP+, soit un gain de $\mathbf{1 1 , 8}$ mois (IC $95 \%$ 0,48-0,94; HR : 0,67; $=0,02)$. 
Il s'agit du premier essai prospectif évaluant la place de la CHIP dans la prise en charge de la carcinose péritonéale d'origine ovarienne en chirurgie d'intervalle, avec des résultats positifs en faveur de la CHIP.

La CHIP n'augmente pas la morbidité post-opératoire, avec un taux d'événements indésirables de grade III-IV similaire dans les deux groupes : $25 \% \mathrm{CHIP}-, 27 \% \mathrm{CHIP}+$, $\mathrm{p}=0,76$, les toxicités les plus fréquentes de grade III-IV étaient les douleurs abdominales, les infections et l'iléus. La néphrotoxicité du Cisplatine a été prévenue de façon efficace par l'administration par intraveineuse de Sodium Thiosulfate, qui inactive le Cisplatine circulant [17]. Cinq patients CHIP+ et trois patients CHIP- ont tout de même présenté des effets indésirables rénaux. Malheureusement, les auteurs ne précisent pas si cette différence est significative, et les conséquences pour ces patientes (degré de l'insuffisance rénale, nécessité d'une dialyse...) Le peu de toxicité, rapporté dans l'essai de van Driel et al. est sujet à caution, et ne préjuge pas de la survenue de toxicité dans d'autres équipes moins encadrées pour réaliser la CHIP.

La CHIP ne modifie pas le délai de reprise de la chimiothérapie (30 jours CHIP- versus 33 jours CHIP+). Cet élément est primordial, puisque la chimiothérapie adjuvante est un pilier de la prise en charge thérapeutique des cancers de l'ovaire avancés.

La qualité de vie était similaire dans les deux groupes. Ce point est assez troublant alors qu'une iléostomie ou une colostomie étaient plus fréquemment réalisées dans le groupe CHIP+ : $72 \%$ versus $43 \%, \mathrm{p}=0,04$. Néanmoins, ces résultats concordent avec ceux de la littérature : l'étude de Chia et coll. a montré que la qualité de vie est préservée en cas de CHIP [18].

L'essai de van Driel et al. a montré que, dans les cancers épithéliaux de stade FIGO III, initialement non résécables, la CHIP réalisée lors de la chirurgie d'intervalle, après trois 
cycles de chimiothérapie intraveineuse, augmente la survie globale et la survie sans rechute (niveau de preuve IB). Ainsi, la CHIP au Cisplatine, avec néphroprotection par Thiosulfate de Sodium intraveineuse, peut être proposée dans les carcinoses ovariennes de stade FIGO III, lors d'une chirurgie d'intervalle [11].

\section{Les critiques de cette étude.}

Il s'agit d'une étude réalisée sur un échantillon de petite taille (245 patientes) présentant une différence entre les groupes pour l'événement «décès » de 15 patientes seulement, soit $6 \%$ de l'effectif global (62\% CHIP- versus $50 \%$ CHIP+; HR : 0,67; p = 0,02). La cause du décès est connue pour cinq patientes (une patiente $\mathrm{CHIP}+$, quatre patientes $\mathrm{CHIP}-$ ), dont trois décès liés à une progression de la pathologie péritonéale. Concernant les dix autres décès, étaient-ils en lien avec la pathologie traitée?

Seules trois patientes par an et par centre ont été incluses dans l'étude. Dix pourcents des patientes (12 dans chaque groupe) avaient une maladie résiduelle inférieure à un centimètre. La répartition des patientes opérées antérieurement est donc équivalente dans les deux groupes. Cependant, la répartition en fonction de la taille du résidu tumoral n'était peut-être pas homogène, alors que l'on sait que la survie est influencée par l'étendue de la maladie résiduelle [19]. Dans l'hypothèse où les résidus tumoraux seraient inférieurs dans le groupe CHIP, alors, le gain en survie pourrait être dû, non pas à l'influence de la CHIP, mais plutôt à l'évolution naturelle d'une maladie moins étendue initialement, donc intrinsèquement plus favorable. En comparaison, l'essai du GOG 132 [20] évaluant la chimiothérapie adjuvante (Cisplatine +/- Paclitaxel) après chirurgie de réduction suboptimale (résidu inférieur à un centimètre), qui représente la situation la plus défavorable, retrouve une survie sans rechute comparable au groupe CHIP + soit 14,1 mois. En revanche la survie générale est meilleure dans le groupe CHIP + de van Driel et al. : 45,7 versus 30,2 mois. 
Dans une lettre à l'éditeur, Vergote dénonce l'absence de stratification selon le type histologique : $87 \%$ des patientes CHIP- versus $92 \%$ CHIP+ présentaient un adénocarcinome séreux de haut grade [21]. En revanche, les groupes étaient déséquilibrés pour les autres types de tumeur, puisque 13 patientes CHIP- présentaient des types histologiques de moins bon pronostic, versus 3 patientes $\mathrm{CHIP}+$ (endométrioide de haut grade, carcinosarcome, mucineux, carcinome à cellules claires, métastases d'une tumeur intestinale), ce qui peut influencer les résultats en faveur du groupe CHIP+.

L'essai de van Driel et al. s'est déroulé dans huit centres. Le centre ayant recruté près de la moitié des patientes (Netherland Cancer Institute, $42 \%$ CHIP-, $43 \%$ CHIP+) est celui dont l'effet de la CHIP est le moins important (HR 0,88 et 0,76 respectivement) et cet effet augmentait pour les centres ayant des faibles effectifs. On pourrait s'attendre, au contraire, à ce que l'effet de la CHIP soit supérieur dans les centres experts ayant un grand volume de patientes, ce qui n'est pas le cas dans cette étude. L'accès aux essais cliniques et/ou aux thérapies ciblées était-il plus important dans le principal centre recruteur, atténuant ainsi potentiellement le bénéfice de la CHIP ?

La randomisation avait lieu avant la chirurgie. Les auteurs ont justifié ce choix par l'organisation du temps opératoire, la préparation des produits de CHIP, et le conditionnement pré-opératoire des patientes. La résection chirurgicale était complète et comparable dans les deux groupes pour $67 \%$ des patientes CHIP- et $69 \%$ des patientes CHIP +.

Les données concernant les sites de rechute pour l'étude de van Driel et al. ne sont pas disponibles. Une étude récente de Ceresoli et al. a évalué les sites de récidive après CHIP : les patientes traitées par chirurgie de cytoréduction seule étaient plus susceptibles de présenter une récidive péritonéale que celles ayant bénéficié d'une CHIP : $43 \%$ CHIP- versus $14 \%$ $\mathrm{CHIP}+[22]$. En revanche la CHIP ne prévenait pas la récidive extra-péritonéale : $30 \%$ CHIP- 
versus $62 \% \mathrm{CHIP}+$. Une récidive péritonéale était associée à un pronostic plus sombre. Ainsi, la CHIP permettrait un contrôle de l'évolution péritonéale et de fait, un gain en survie. La durée moyenne d'hospitalisation était augmentée de deux jours en cas de CHIP (huit jours CHIP- versus dix jours CHIP+, $\mathrm{p}>0,005)$, dont un jour en unité de soins intensifs comme le recommande le protocole. En considérant les frais liés à l'hospitalisation et aux complications potentielles, la CHIP est deux fois plus couteuse qu'un recours au Bevacizumab et trois fois plus qu'une chirurgie d'intervalle [23].

\section{Autres indications potentielles de la CHIP.}

En première intention, la CHIP permettrait de traiter précocement les résidus microscopiques avant l'apparition d'un profil de chimiorésistance aux sels de platine [14]. Néanmoins, se pose la question du délai de l'introduction de la chimiothérapie adjuvante, notamment en cas de complications spécifiques de la CHIP: insuffisance rénale résiduelle au Cisplatine ou fistule digestive. En traitement de consolidation, la CHIP impose un second look chirurgical, mais permettrait de mettre en évidence une récidive précoce ou une chimiorésistance primaire aux sels de platine, qui est une situation de mauvais pronostic [3]. En 2010 l'essai français CHIPOVAC testant la CHIP à l'Oxaliplatine en consolidation a été interrompu prématurément pour toxicité majeure : neuf des vingt-huit patientes incluses ont présenté un hémopéritoine nécessitant une reprise chirurgicale en urgence [24]. Dans l'analyse rétrospective de Mendivil et al. la CHIP en consolidation (Carboplatine) allongerait la survie sans rechute de cinq mois en comparaison à une chimiothérapie adjuvante $(25,1$ versus 20 mois, $\mathrm{p}=0,024)$, mais sans impact sur la survie générale [25]. Actuellement, aucune étude prospective évaluant la CHIP au Cisplatine en chirurgie initiale ou en traitement de consolidation n'est disponible. 
En situation de rechute, le pronostic est dominé par la chimiosensibilité aux sels de platine. Les patientes réfractaires à la chimiothérapie et les patientes platine-résistantes (délai entre la dernière administration de sels de platine et la rechute inférieure à six mois) ont un pronostic très réservé avec une survie moyenne de neuf mois [26]. Dans ce cas la chirurgie n'est pas justifiée, ce qui explique que la CHIP n'ait pas été évaluée pour ce groupe de patientes. Les patientes «platine-sensibles » (délai entre la dernière administration de sels de platine et la rechute supérieure à six mois) ont un pronostic bien meilleur avec une survie globale de trois ans [26]. L'étude DESKTOP III présentée à l'ASCO en 2017 a montré un bénéfice substantiel pour la survie sans rechute de 5,6 mois pour les patientes opérées à la rechute (14 mois versus 19,6 mois, p < 0,001), uniquement dans le cas où la résection était complète [27]. L'étendue de la carcinose via le PCI était un facteur prédictif d'échec de résection R0. Cependant, l'étude GOG 213 a retrouvé le même bénéfice de survie sans rechute mais sans bénéfice en termes de survie générale à réaliser une chirurgie dans cette indication, même chez les patientes sans reliquat $(\mathrm{HR} 1,11)$ [28]. Il y a même une tendance à l'aggravation du pronostic chez les patientes opérées par rapport aux patientes non opérées. Les critères d'éligibilité à la chirurgie étaient cependant moins rigoureux que ceux de l'essai DESKTOP III. Si la chirurgie est challengée dans cette indication, la place de la CHIP, imposant un résidu tumoral millimétrique, est-elle alors justifiée ?

Cependant, le bénéfice de la CHIP à la rechute a été étudié dans de nombreuses cohortes rétrospectives. La méta-analyse de Chiva et al., incluant 499 patientes en rechute platinesensible ayant bénéficié d'une chirurgie de cytoréduction $(71 \% \mathrm{CC} 0)+$ CHIP retrouve une survie générale de 36,5 mois et une survie sans rechute de 20 mois [29]. Rappelons que dans l'étude DESKTOP I la survie sans rechute des patientes opérées à la rechute était de 19,6 mois [30]. L'intérêt de la CHIP paraît donc modeste. 
L'association d'une chirurgie de cytoréduction rigoureuse, à un traitement éradiquant une maladie résiduelle microscopique, à l'origine d'inévitables récidives, reste une option séduisante dont l'évaluation doit se poursuivre au sein d'essais de phase III. CHIPOR évalue l'efficacité de la CHIP dans le traitement de la première récidive du cancer de l'ovaire chez des patientes platine-sensible [31].

\section{D'autres études cliniques récentes nuancent l'intérêt de la CHIP.}

Indépendamment des critiques potentielles de l'étude de van Driel et al., le message concernant la CHIP est troublé par plusieurs éléments. Tout d'abord, la chimiothérapie par voie intrapéritonéale est remise en question avec les résultats du GOG 252 dans lequel l'administration intrapéritonéale en comparaison à la voie intraveineuse, associée à du Bevacizumab, n'augmente pas la survie sans rechute, quelle que soit la taille du résidu tumoral après chirurgie de cytoréduction : survie sans rechute 31,3 mois (intraveineuse) versus 31,8 mois (intrapéritonéale Carboplatine) versus 33,8 mois (intrapéritonéale Cisplatine) [32]. Ces résultats annihilent le rationnel de la voie intrapéritonéale.

D'autre part, deux études prospectives retrouvent des résultats contradictoires à ceux de van Driel et al. Premièrement, les résultats intermédiaires de l'étude de Lim et al., réalisée selon le même design que l'étude de van Driel et al., et présentée à l'ASCO en 2017, sont négatifs [15]. Cependant des patientes de stade FIGO IV initial ont été randomisées. Deuxièmement, l'étude PRODIGE 7 visant à évaluer le rôle de la CHIP à l'Oxaliplatine, après chirurgie de cytoréduction complète pour le traitement de la carcinose péritonéale d'origine colorectale, ne retrouve pas de différence statistiquement significative sur la survie générale et la survie sans rechute : survie sans rechute 11,1 mois $\mathrm{CHIP}$ - versus 13,1 mois $\mathrm{CHIP}+, \mathrm{p}=0,486$; survie générale 41,2 mois CHIP- versus 41,7 mois CHIP+, p=0,995 [33]. À 60 jours, le taux d'effet indésirables de grade III-V était plus élevé dans le groupe CHIP+ (24,1 versus 13,6\%, 
$\mathrm{p}=0,03)$. Les résultats de PRODIGE 7 invitent à la réflexion, avec un changement des pratiques pour les carcinoses péritonéales d'origine colorectale.

Enfin, l'avènement des thérapies ciblées occulte l'intérêt de l'approche thérapeutique intrapéritonéale. S'il est aisé de penser que l'éradication de la maladie microscopique est facilitée par l'absence de résidu tumoral après chirurgie de cytoréduction, la survie des patientes dépend aujourd'hui des traitements systémiques adjuvants. L'enjeu thérapeutique actuel consiste à déterminer la meilleure combinaison thérapeutique, et il reste à savoir si la CHIP peut s'associer à ces nouvelles thérapies : anti-angiogéniques, anti-PD1 et inhibiteur des PARP.

L'essai GOG 0218 publié par Burger et al. en 2011 montre que l'ajout du Bevacizumab à une chimiothérapie adjuvante après chirurgie de cytoréduction prolonge significativement la survie sans rechute de quatre mois (sans impact sur la survie globale) [34]. En traitement néoadjuvant, les études ANTHALYA et MITO 16 ont montré que le Bevacizumab facilite la chirurgie de cytoréduction complète sans augmentation de la morbidité [35,36]. Deux essais de phase I et II ont démontré la faisabilité de l'association Bevacizumab en séquentiel avec la CHIP $[37,38]$.

L'utilisation des anti-PARP est une révolution dans le traitement des cancers de l'ovaire au même titre que fut celle des sels de platine. L'Olaparib a fait la preuve de son bénéfice en traitement d'entretien chez les patientes mutées BRCA platine-sensible en première ligne, puisqu'il diminue le risque de récidive à trois ans de $70 \%$ (27\% groupe Olaparib versus $60 \%$ groupe placebo, $\mathrm{HR}=0,3$; IC $95 \%$ 0,23-0,41; $<<0,001)$ [39], et en situation de rechute puisqu'il prolonge la survie sans rechute de 13,6 mois (survie sans rechute 19,1 mois groupe Olaparib versus 5,5 mois groupe placebo, $\mathrm{HR}=0,30, \mathrm{p}<0,0001$ ) [40]. Il est probable que les anti-PARP pourraient offrir un bénéfice au-delà des tumeurs portant une mutation BRCA 
[41]. Un essai de phase III a montré un bénéfice de l'utilisation du Niraparib chez les patientes en rechute platine-sensible, même dans le sous-groupe non BRCA muté.

Enfin, l'hypoxie sensibilisant les cellules tumorales aux anti-PARP, l'ajout d'un antiangiogénique pourrait augmenter l'activité de l'anti-PARP. Un essai clinique combinant l'Olaparib a un anti-angiogénique (Cédiranib) chez des patientes avec un cancer de l'ovaire en rechute platine-sensible a montré une augmentation de la survie sans rechute : 17,7 versus 9 mois, $\mathrm{p}=0,005$. On attend également les résultats de l'essai PAOLA, évaluant Olaparib versus placebo en première ligne, chez toutes les patientes de stade FIGO III-IV, mutées ou non mutées, en association avec le Bevacizumab en traitement d'entretien [42].

Enfin, pour les patientes non résécables d'emblée, plusieurs essais de phase III visant à évaluer les immunomodulateurs sont en cours [43]. L’efficacité est évaluée par la qualité de la résection en chirurgie intervallaire.

\section{Conclusion.}

La CHIP permet-elle d'améliorer la survie des patientes présentant une carcinose péritonéale d'origine ovarienne ? La preuve de ce bénéfice reste à démontrer.

Actuellement, 8 essais randomisés évaluant la CHIP dans le cancer de l'ovaire sont en cours [44]. Les résultats présentés par van Driel et al. sont encourageants, cette étude est la première ayant démontré un rôle favorable de la CHIP en chirurgie d'intervalle. Cependant, ces résultats ne permettent pas de faire de la CHIP un traitement standard. Avec l'émergence des thérapies ciblées, la place de la CHIP reste à définir.

Dans l'attente des résultats de l'essai français CHIPOR notamment, le recours à la CHIP doit se faire en centre hyper-spécialisé, et devrait idéalement être encadré par un essai clinique. 


\section{RÉFÉRENCES BIBLIOGRAPHIQUES.}

[1]InfoCancer: Epidémiologie des cancers féminins - Cancer de l'ovaire. WwwInfocancerRg n.d.

[2]Kurman R, Carcangiu M, Herrington C, Young R. WHO Classification of Tumours of Female Reproductive Organs. In WHO Classification of Tumours. A. Aufl. 2014.

[3]Ferron G, Martinez A, Mery E, Querleu D, Thomas F, Chatelut L, et al. Collège National des Gynécologues et Obstétriciens Français. Journal de Gynécologie Obstétrique et Biologie de la Reproduction 2010;34:513.

[4]Cotte E, Colomban O, Guitton J, Tranchand B, Bakrin N, Gilly F-N, et al. Population Pharmacokinetics and Pharmacodynamics of Cisplatinum During Hyperthermic Intraperitoneal Chemotherapy Using a Closed Abdominal Procedure. The Journal of Clinical Pharmacology 2011;51:9-18.

[5]Markman M. Intraperitoneal therapy for treatment of malignant disease principally confined to the peritoneal cavity. Crit Rev Oncol Hematol 1993;14:15-28.

[6]Armstrong DK, Bundy B, Wenzel L, Huang HQ, Baergen R, Lele S, et al. Intraperitoneal Cisplatin and Paclitaxel in Ovarian Cancer. New England Journal of Medicine 2006;354:3443.

[7]Alberts DS, Liu PY, Hannigan EV, O’Toole R, Williams SD, Young JA, et al. Intraperitoneal cisplatin plus intravenous cyclophosphamide versus intravenous cisplatin plus intravenous cyclophosphamide for stage III ovarian cancer. N Engl J Med 1996;335:1950-5.

[8]Markman M, Bundy BN, Alberts DS, Fowler JM, Clark-Pearson DL, Carson LF, et al. Phase III Trial of Standard-Dose Intravenous Cisplatin Plus Paclitaxel Versus Moderately High-Dose Carboplatin Followed by Intravenous Paclitaxel and Intraperitoneal Cisplatin in Small-Volume Stage III Ovarian Carcinoma: An Intergroup Study of the Gynecologic Oncology Group, Southwestern Oncology Group, and Eastern Cooperative Oncology Group. JCO 2001;19:1001-7.

[9]Garcia-Soto AE, Java JJ, Nieves Neira W, Pearson JM, Cohn DE, Lele SB, et al. Does time interval between surgery and intraperitoneal chemotherapy administration in advanced ovarian cancer carry a prognostic impact? An NRG Oncology/Gynecologic Oncology Group study ancillary study. Gynecologic Oncology 2016;143:484-9.

[10]Jaaback K, Johnson N, Lawrie TA. Intraperitoneal chemotherapy for the initial management of primary epithelial ovarian cancer. Cochrane Database Syst Rev 2016:CD005340.

[11]Institut National du Cancer. Thésaurus: Conduites à tenir initiales devant des patientes atteintes d'un cancer épithélial de l'ovaire. 2018.

[12]Muller M, Chérel M, Dupré P-F, Gouard S, Collet M, Classe J-M. Cytotoxic effect of 
hyperthermia and chemotherapy with platinum salt on ovarian cancer cells: results of an in vitro study. Eur Surg Res 2011;46:139-47.

[13]Witkamp AJ, de Bree E, Van Goethem R, Zoetmulder FA. Rationale and techniques of intra-operative hyperthermic intraperitoneal chemotherapy. Cancer Treatment Reviews 2001;27:365-74.

[14]Bakrin N, Classe J-M, Pomel C, Gouy S, Chene G, Glehen O. La chimiohyperthermie intrapéritonéale (CHIP) dans les cancers ovariens. Journal de Chirurgie Viscérale 2014;151:358-65.

[15]Lim M, Suk-Joon C, Yoo H. Randomized trial of hyperthermic intraperitoneal chemotherapy (HIPEC) in women with primary advanced peritoneal, ovarian, and tubal cancer 2017.

[16]van Driel WJ, Koole SN, Sikorska K, Schagen van Leeuwen JH, Schreuder HWR, Hermans RHM, et al. Hyperthermic Intraperitoneal Chemotherapy in Ovarian Cancer. New England Journal of Medicine 2018;378:230-40. doi:10.1056/NEJMoa1708618.

[17]Howell SB, Pfeifle CL, Wung WE, Olshen RA, Lucas WE, Yon JL, et al. Intraperitoneal cisplatin with systemic thiosulfate protection. Ann Intern Med 1982;97:845-51.

[18]Chia CS, Tan WJ, Wong JFS, Tan GHC, Lim C, Wang W, et al. Quality of life in patients with peritoneal surface malignancies after cytoreductive surgery and hyperthermic intraperitoneal chemotherapy. Eur J Surg Oncol 2014;40:909-16.

[19]Bakrin N, Bereder JM, Decullier E, Classe JM, Msika S, Lorimier G, et al. Peritoneal carcinomatosis treated with cytoreductive surgery and Hyperthermic Intraperitoneal Chemotherapy (HIPEC) for advanced ovarian carcinoma: A French multicentre retrospective cohort study of 566 patients. European Journal of Surgical Oncology (EJSO) 2013;39:143543.

[20]Muggia FM, Braly PS, Brady MF, Sutton G, Niemann TH, Lentz SL, et al. Phase III randomized study of cisplatin versus paclitaxel versus cisplatin and paclitaxel in patients with suboptimal stage III or IV ovarian cancer: a gynecologic oncology group study. J Clin Oncol 2000;18:106-15.

[21]Hyperthermic Intraperitoneal Chemotherapy in Ovarian Cancer. N Engl J Med. 2018 Apr 5;378(14):1362-4. doi:10.1056/NEJMc1802033.

[22]Ceresoli M, Verrengia A, Montori G, Busci L, Coccolini F, Ansaloni L, et al. Effect of cytoreductive surgery and hyperthermic intraperitoneal chemotherapy on relapse pattern in primary epithelial ovarian cancer: a propensity score based case-control study. Journal of Gynecologic Oncology 2018;29.

[23]Behbakht K, Cohn DE, Straughn JM. Hyperthermic intraperitoneal chemotherapy (HIPEC) is cost-effective in the management of primary ovarian cancer. Gynecologic Oncology 2018;151:4-5.

[24]Pomel C, Ferron G, Lorimier G, Rey A, Lhomme C, Classe JM, et al. Hyperthermic intraperitoneal chemotherapy using oxaliplatin as consolidation therapy for advanced epithelial ovarian carcinoma. Results of a phase II prospective multicentre trial. CHIPOVAC study. Eur 
J Surg Oncol 2010;36:589-93.

[25]Mendivil AA, Rettenmaier MA, Abaid LN, Brown JV, Mori KM, Lopez KL, et al. Consolidation hyperthermic intraperitoneal chemotherapy for the treatment of advanced stage ovarian carcinoma: a 3 year experience. Cancer Chemotherapy and Pharmacology 2017;80:405-10.

[26]Selle F, Burcoveanu D, Cortez A, Rouzier R, Bazot M, Dubot C. Chimiothérapie des récidives des cancers de l'ovaire. Medecine Therapeutique Medecine de la Reproduction, Gynecologie et Endocrinologie 2012;14:51-62 suppl 1.

[27]Du Bois A, Vergote I, Ferron I, Reuss A, Meier W, Greggi S. Randomized controlled phase III study evaluating the impact of secondary cytoreductive surgery in recurrent ovarian cancer: AGO DESKTOP III/ENGOT ov20. J Clin Oncol 2017;35:Abstract 5501.

[28]Coleman RL, Brady MF, Herzog TJ, Sabbatini P, Armstrong DK, Walker JL, et al. Bevacizumab and paclitaxel-carboplatin chemotherapy and secondary cytoreduction in recurrent, platinum-sensitive ovarian cancer (NRG Oncology/Gynecologic Oncology Group study GOG-0213): a multicentre, open-label, randomised, phase 3 trial. Lancet Oncol 2017;18:779-91.

[29]Chiva L, Lapuente F, Castellanos T, Alonso S, Gonzalez-Martin A. What Should We Expect After a Complete Cytoreduction at the Time of Interval or Primary Debulking Surgery in Advanced Ovarian Cancer? Annals of Surgical Oncology 2016;23:1666-73.

[30]Harter P, du Bois A, Hahmann M, Hasenburg A, Burges A, Loibl S, et al. Surgery in recurrent ovarian cancer: the Arbeitsgemeinschaft Gynaekologische Onkologie (AGO) DESKTOP OVAR trial. Ann Surg Oncol 2006;13:1702-10.

[31]Hyperthermic Intra-Peritoneal Chemotherapy (HIPEC) in Relapse Ovarian Cancer Treatment - CHIPOR - ClinicalTrials.gov n.d.

https://clinicaltrials.gov/ct2/show/NCT01376752 (accessed October 29, 2018).

[32]Walker J, Brady MF, DiSilvestro PA, Fujiwara K, Alberts D, Zheng W, et al. A phase III trial of bevacizumab with IV versus IP chemotherapy for ovarian, fallopian tube, and peritoneal carcinoma: An NRG Oncology Study. Gynecologic Oncology 2016;141:208..

[33]Quenet F. Meeting Library | A UNICANCER phase III trial of hyperthermic intraperitoneal chemotherapy (HIPEC) for colorectal peritoneal carcinomatosis (PC): PRODIGE 7. n.d. https://meetinglibrary.asco.org/record/158740/abstract (accessed September 11, 2018).

[34]Burger RA, Brady MF, Bookman MA, Fleming GF, Monk BJ, Huang H, et al. Incorporation of bevacizumab in the primary treatment of ovarian cancer. N Engl J Med 2011;365:2473-83.

[35]Rouzier R, Gouy S, Selle F, Lambaudie E, Floquet A, Fourchotte V, et al. Efficacy and safety of bevacizumab-containing neoadjuvant therapy followed by interval debulking surgery in advanced ovarian cancer: Results from the ANTHALYA trial. Eur J Cancer 2017;70:133-42.

[36]Daniele G, Lorusso D, Scambia G, Cecere SC, Nicoletto MO, Breda E, et al. Feasibility and outcome of interval debulking surgery (IDS) after carboplatin-paclitaxel-bevacizumab 
(CPB): A subgroup analysis of the MITO-16A-MaNGO OV2A phase 4 trial. Gynecol Oncol 2017;144:256-9.

[37]Paris I, Cianci S, Vizzielli G, Fagotti A, Ferrandina G, Gueli Alletti S, et al. Upfront HIPEC and bevacizumab-containing adjuvant chemotherapy in advanced epithelial ovarian cancer. Int J Hyperthermia 2018:1-5.

[38]Gouy S, Ferron G, Glehen O, Bayar A, Marchal F, Pomel C, et al. Results of a multicenter phase I dose-finding trial of hyperthermic intraperitoneal cisplatin after neoadjuvant chemotherapy and complete cytoreductive surgery and followed by maintenance bevacizumab in initially unresectable ovarian cancer. Gynecologic Oncology 2016;142:23742.

[39]Moore K, Colombo N, Scambia G, Kim B-G, Oaknin A, Friedlander M, et al.

Maintenance Olaparib in Patients with Newly Diagnosed Advanced Ovarian Cancer. N Engl J Med. 2018 Dec 27;379(26):2495-2505

[40]Pujade-Lauraine E, Ledermann JA, Selle F, Gebski V, Penson RT, Oza AM, et al. Olaparib tablets as maintenance therapy in patients with platinum-sensitive, relapsed ovarian cancer and a BRCA1/2 mutation (SOLO2/ENGOT-Ov21): a double-blind, randomised, placebo-controlled, phase 3 trial. Lancet Oncol 2017;18:1274-84.

[41]Auguste A, Leary A. [Abnormalities of DNA repair and gynecological cancers]. Bull Cancer. 2017 Nov;104(11):971-980

[42]Platine, Avastin and OLAparib in 1st Line - Full Text View - ClinicalTrials.gov n.d. https://clinicaltrials.gov/ct2/show/NCT02477644 (accessed October 29, 2018).

[43]PEMBRO With Chemo in Neo Adj Treatment of Ovarian Cancer . - Full Text View ClinicalTrials.gov n.d. https://clinicaltrials.gov/ct2/show/NCT03275506 (accessed October 29, 2018).

[44]Iavazzo C, Spiliotis J. Hyperthermic intraperitoneal chemotherapy (HIPEC) for ovarian cancer: a "useless intraoperative fever" or the next hot voice in the surgical management of the "silent killer"? Arch Gynecol Obstet. 2018 Oct;298(4):673-674. 


\begin{tabular}{|c|c|c|c|c|c|c|c|c|c|c|}
\hline & $\begin{array}{l}\text { Nom de } \\
\text { l'étude }\end{array}$ & $\begin{array}{l}\text { Auteur } \\
\text { principal }\end{array}$ & $\begin{array}{l}\text { Année de } \\
\text { publication }\end{array}$ & $\begin{array}{l}\text { Type } \\
\text { d'étude }\end{array}$ & Population cible & Modalités de l'étude & $\begin{array}{l}\text { Nombre de } \\
\text { sujets inclus }\end{array}$ & Objectif principal & Conclusion des auteurs & Effets indésirables \\
\hline \multirow{8}{*}{ IP } & $\begin{array}{l}\text { GOG } \\
114\end{array}$ & Markman & 1993 & $\begin{array}{l}\text { Essai clinique } \\
\text { randomisé }\end{array}$ & $\begin{array}{l}\text { Carcinose péritonéale ovarienne de } \\
\text { stade FIGO III, après chirurgie de } \\
\text { résection complète (résidu tumoral < } \\
1 \mathrm{~cm} \text { ). }\end{array}$ & $\begin{array}{l}\text { Randomisation en } 2 \text { bras : } \\
\text {-bras } 1 \text { : IV Paclitaxel + Cisplatine } \\
\text {-bras } 2 \text { : IV Carboplatine + Paclitaxel + IP Cisplatine }\end{array}$ & 523 & Évaluation de la SG. & $\begin{array}{l}\text { Augmentation de la SG de } \\
10,7 \text { mois et de la SSR de } \\
5,1 \text { mois dans le bras IP. }\end{array}$ & $\begin{array}{l}\text { Plus d'effets indésirables } \\
\text { de grade III-IV dans le } \\
\text { groupe IP (neurotoxicité, } \\
\text { hématotoxicité). }\end{array}$ \\
\hline & $\begin{array}{l}\text { GOG } \\
104\end{array}$ & Alberts & 1996 & $\begin{array}{l}\text { Essai clinique } \\
\text { randomisé }\end{array}$ & $\begin{array}{l}\text { Carcinose péritonéale ovarienne de } \\
\text { stade FIGO III, après chirurgie de } \\
\text { résection suboptimale (résidu tumoral } \\
<2 \mathrm{~cm} \text { ). }\end{array}$ & $\begin{array}{l}\text { Randomisation en } 2 \text { bras: } \\
\text {-bras } 1 \text { : IV Cyclophosphamide + Cisplatine } \\
\text {-bras } 2 \text { : IV Cyclophosphamide + IP Cisplatine }\end{array}$ & 654 & Évaluation de la SG. & $\begin{array}{l}\text { Augmentation de la SG de } \\
8 \text { mois dans le bras IP. }\end{array}$ & $\begin{array}{l}\text { Plus d'effets indésirables } \\
\text { de grade III-IV dans le } \\
\text { groupe IV. }\end{array}$ \\
\hline & EORTC & Piccart & 1997 & $\begin{array}{l}\text { Essai clinique } \\
\text { randomisé }\end{array}$ & $\begin{array}{l}\text { Carcinose péritonéale ovarienne de } \\
\text { stade FIGO III, après chirurgie de } \\
\text { résection suboptimale ou optimale. }\end{array}$ & $\begin{array}{l}\text { Randomisation en } 2 \text { bras : } \\
\text {-bras } 1 \text { : IV Cyclophosphamide + Cisplatine } \\
\text {-bras } 2 \text { : IV Paclitaxel + Cisplatine } \\
\text { Puis second look en cas de réponse clinique } \\
\text { complète. }\end{array}$ & 680 & Évaluation de la SSR. & $\begin{array}{l}\text { Augmentation de la SSR } \\
\text { de } 4,6 \text { mois dans le bras } \\
\text { avec Paclitaxel, quel que } \\
\text { soit le volume tumoral } \\
\text { résiduel après chirurgie } \\
\text { première. }\end{array}$ & $\begin{array}{l}\text { Toxicité neurologique de } \\
\text { grade III-IV plus } \\
\text { importantes avec le } \\
\text { Paclitaxel. Toxicité } \\
\text { digestive plus importante } \\
\text { avec le } \\
\text { Cyclophosphamide. }\end{array}$ \\
\hline & $\begin{array}{l}\text { GOG } \\
132\end{array}$ & Muggia & 2000 & $\begin{array}{l}\text { Essai clinique } \\
\text { randomisé }\end{array}$ & $\begin{array}{l}\text { Carcinose péritonéale ovarienne de } \\
\text { stade FIGO III, après chirurgie de } \\
\text { résection suboptimale (résidu tumoral } \\
<1 \mathrm{~cm} \text { ). }\end{array}$ & $\begin{array}{l}\text { Randomisation en } 3 \text { bras : } \\
\text {-bras } 1 \text { : IV Cisplatine } \\
\text {-bras } 2 \text { : IV Paclitaxel } \\
\text {-bras } 3 \text { : IV Paclitaxel + Cisplatine }\end{array}$ & 648 & $\begin{array}{l}\text { Évaluation de la SSR et } \\
\text { de la SG. }\end{array}$ & $\begin{array}{l}\text { Augmentation de la SSR } \\
\text { dans les groupes } \\
\text { comprenant du } \\
\text { Cisplatine. Les médianes } \\
\text { de SG sont similaires dans } \\
\text { les } 3 \text { groupes. }\end{array}$ & $\begin{array}{l}\text { Toxicité de grades III ou } \\
\text { IV digestives, rénales, } \\
\text { neurologiques, } \\
\text { hématologiques plus } \\
\text { fréquentes avec } \\
\text { Cisplatine seul. }\end{array}$ \\
\hline & $\begin{array}{l}\text { GOG } \\
172\end{array}$ & Amstrong & 2006 & $\begin{array}{l}\text { Essai clinique } \\
\text { randomisé }\end{array}$ & $\begin{array}{l}\text { Carcinose péritonéale de stade FIGO } \\
\text { III, avec résection chirurgicale } \\
\text { première complète (résidu tumoral < } \\
1 \mathrm{~cm} \text { ). }\end{array}$ & $\begin{array}{l}\text { Randomisation en } 2 \text { bras : } \\
\text {-bras } 1 \text { : IV Cisplatine + Paclitaxel } \\
\text {-bras } 2 \text { : IV Paclitaxel + IP Cisplatine + IP Paclitaxel }\end{array}$ & 429 & $\begin{array}{l}\text { Évaluation de la SG et } \\
\text { SSR. }\end{array}$ & $\begin{array}{l}\text { Augmentation de la SSR } \\
\text { de } 5,3 \text { mois et de la SG de } \\
15,9 \text { mois dans le bras IP. }\end{array}$ & $\begin{array}{l}\text { Plus d'effets indésirables } \\
\text { de grade III-IV dans le } \\
\text { groupe IP }(p<0,001)\end{array}$ \\
\hline & $\begin{array}{l}\text { GOG } \\
218\end{array}$ & Burger & 2011 & $\begin{array}{l}\text { Essai clinique } \\
\text { randomisé }\end{array}$ & $\begin{array}{l}\text { Carcinose péritonéale ovarienne de } \\
\text { stade FIGO III, après chirurgie de } \\
\text { résection complète. }\end{array}$ & $\begin{array}{l}\text { Randomisation en } 3 \text { bras : } \\
\text {-bras } 1 \text { : IV Paclitaxel + Carboplatine } \\
\text {-bras } 2 \text { : IV Paclitaxel + Carboplatine + } \\
\text { Bévacizumab } \\
\text {-bras } 3 \text { : même schéma que le bras } 2 \text { mais avec un } \\
\text { traitement d'entretien par Bévacizumab }\end{array}$ & 1873 & Évaluation de la SSR. & $\begin{array}{l}\text { Augmentation de la SSR } \\
\text { de } 4 \text { mois dans le groupe } \\
\text { avec traitement } \\
\text { d'entretien par } \\
\text { Bévacizumab. }\end{array}$ & $\begin{array}{l}\text { Plus d'hypertension chez } \\
\text { les patients traités avec } \\
\text { du Bévacizumab. }\end{array}$ \\
\hline & $\begin{array}{l}\text { GOG } \\
252\end{array}$ & Walker & 2016 & $\begin{array}{l}\text { Essai clinique } \\
\text { randomisé }\end{array}$ & $\begin{array}{l}\text { Carcinose péritonéale d'origine } \\
\text { ovarienne de stade FIGO III, après } \\
\text { résection chirurgicale première } \\
\text { optimale (résection chirurgicale } \\
\text { complète : } 57 \% \text { ). }\end{array}$ & $\begin{array}{l}\text { Les patientes éligibles recevaient du Bévacizumab, } \\
\text { randomisation en } 3 \text { groupes : } \\
\text {-bras } 1 \text { : IV Carboplatine+Paclitaxel } \\
\text {-bras } 2 \text { : IP Carboplatine + IV Paclitaxel } \\
\text {-bras } 3 \text { : IV Paclitaxel + IP Cisplatine + IP Paclitaxel }\end{array}$ & 1560 & Évaluation de la SSR. & $\begin{array}{l}\text { Pas de différence de SSR } \\
\text { entre les trois groupes. }\end{array}$ & $\begin{array}{l}\text { Toxicité neurologique } \\
\text { majoritairement, de } \\
\text { grade III, identique dans } \\
\text { les } 3 \text { groupes }\end{array}$ \\
\hline & $\begin{array}{l}\text { GOG } \\
0213\end{array}$ & Coleman & 2017 & $\begin{array}{l}\text { Essai clinique } \\
\text { randomisé }\end{array}$ & $\begin{array}{l}\text { Carcinose péritonéale d'origine } \\
\text { ovarienne, en récidive, platine } \\
\text { sensible, ayant reçu en première ligne } \\
\text { et/ou en maintenance du } \\
\text { Bevacizumab. }\end{array}$ & $\begin{array}{l}\text { Randomisation en } 2 \text { groupes: } \\
\text {-bras } 1 \text { : chimiothérapie seule Carboplatine + } \\
\text { Paclitaxel } \\
\text {-bras } 2 \text { : chimiothérapie + Bevacizumab. } \\
\text { +/- associé à une 2è chirurgie d'exérèse. }\end{array}$ & 674 & $\begin{array}{l}\text { Évaluer la SG et la } \\
\text { tolérance de la reprise } \\
\text { du Bevacizumab, et } \\
\text { l'intérêt d'une 2è cyto- } \\
\text { réduction. }\end{array}$ & $\begin{array}{l}\text { Pas de différence de SG } \\
\text { entre les groupes. } \\
\text { Tendance à l'amélioration } \\
\text { de la SSR mais sans } \\
\text { impact sur la SG, si 2è } \\
\text { cyto-réduction. }\end{array}$ & $\begin{array}{l}\text { Effets indésirable de } \\
\text { grade III-IV similaires } \\
\text { dans les } 2 \text { groupes. }\end{array}$ \\
\hline \multirow[t]{2}{*}{ CHIP } & $\begin{array}{l}\text { Prodige } \\
7\end{array}$ & Quenet & 2018 & $\begin{array}{l}\text { Essai clinique } \\
\text { randomisé }\end{array}$ & $\begin{array}{l}\text { Carcinose péritonéale d'origine } \\
\text { colorectale de stade IV. }\end{array}$ & $\begin{array}{l}\text { Chirurgie intervallaire, randomisation après } \\
\text { résection chirurgicale complète : CHIP à } \\
\text { I'Oxaliplatine ou chirurgie seule. }\end{array}$ & 265 & $\begin{array}{l}\text { Évaluation de la survie } \\
\text { globale. }\end{array}$ & $\begin{array}{l}\text { Pas de différence de } \\
\text { survie entre les deux } \\
\text { bras, en SG ou en SSR } \\
\text { (médiane de survie : } 41,7 \\
\text { mois CHIP+ versus } 41,2 \\
\text { mois CHIP-, NS). }\end{array}$ & $\begin{array}{l}\text { Morbi-mortalité } \\
\text { équivalente à } 30 \text { jours, } \\
\text { surmorbidité dans le bras } \\
\text { CHIP à } 60 \text { jours. }\end{array}$ \\
\hline & OVHIPEC & van Driel & 2018 & $\begin{array}{l}\text { Essai clinique } \\
\text { randomisé }\end{array}$ & $\begin{array}{l}\text { Carcinose péritonéale d'origine } \\
\text { ovarienne ou tubaire de stade FIGO } \\
\text { III, non résécable d'emblée. Chirurgie } \\
\text { d'intervalle après } 3 \text { cures de } \\
\text { chimiotérapie. }\end{array}$ & $\begin{array}{l}\text { Chirurgie intervallaire, randomisation après } \\
\text { résection chirurgicale complète: CHIP à base de } \\
\text { Cisplatine ou chirurgie seule. }\end{array}$ & 245 & $\begin{array}{l}\text { Évaluation de la survie } \\
\text { sans récidive. }\end{array}$ & $\begin{array}{l}\text { Augmentation de la SSR } \\
\text { de } 3,5 \text { mois et de la } S G \text { de } \\
11,8 \text { mois dans le bras } \\
\text { CHIP. }\end{array}$ & $\begin{array}{l}\text { Taux d'évènements } \\
\text { indésirables de grade III- } \\
\text { IV similaire dans les deux } \\
\text { groupes. }\end{array}$ \\
\hline
\end{tabular}

